\title{
Trials in developing a nanoscale material for extravascular contrast-enhanced ultrasound targeting hepatocellular carcinoma
}

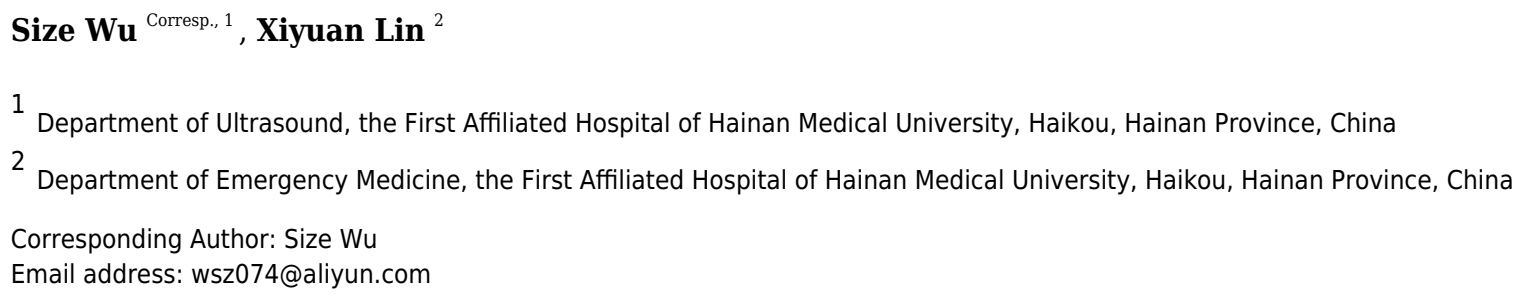

Background. Medical imaging is an important approach for the diagnosis of hepatocellular carcinoma (HCC), a common life threaten disease, however, the diagnostic efficiency is still not optimal. Developing a novel method to improve diagnosis is necessary. The aim of this project was to formulate a material that can combine with GPC3 of HCC for targeted enhanced ultrasound. Methods. A material of sulfur hexafluoride $\left(\mathrm{SF}_{6}\right)$ filled liposome microbubbles and conjugated with synthesized peptide (LSPMbs) was prepared and assessed in vitro and vivo. Liposome microbubbles were made of DPPC, DPPG, DSPE-PEG2000, and $\mathrm{SF}_{6}$, using thin film method to form shell, followed filling $\mathrm{SF}_{6}$, and conjugating peptide. A carbodiimide method was used for covalent conjugation of peptide to LSMbs. Results. The prepared LSPMbs appeared round shaped, with size of $380.9 \pm 176.5 \mathrm{~nm}$, and Zeta potential of $-51.4 \pm 10.4 \mathrm{mV}$. LSPMbs showed high affinity to Huh-7 cells in vitro, presented good enhanced ultrasound effects, did not show cytotoxicity, and did not exhibit targeted fluorescence and enhanced ultrasound in animal xenograft tumors. Conclusion. Extravascular contrast-enhanced ultrasound targeted GPC3 on HCC may not be realized, and the reason may be that targeted contrast agents of microbubbles are hard to access and accumulate in the tumor stroma and matrix. 
1 Title: Trials in developing a nanoscale material for extravascular contrast-enhanced

\section{2 ultrasound targeting hepatocellular carcinoma}

3 Size $\mathrm{Wu}^{1}$, Xiyuan $\operatorname{Lin}^{2}$

$4{ }^{1}$ Department of Ultrasound, the First Affiliated Hospital of Hainan Medical University, Haikou,

5 Hainan Province, China

$6 \quad{ }^{2}$ Department of Emergency Medicine, the First Affiliated Hospital of Hainan Medical

7 University, Haikou, Hainan Province, China

8 Corresponding Author:

$9 \quad$ Size $\mathrm{Wu}^{1}$

10 No.31, Longhua Road, Haikou, Hainan Province, 570102, China

11 Email address: wsz074@aliyun.com

\section{Abstract}

14 Background. Medical imaging is an important approach for the diagnosis of hepatocellular carcinoma (HCC), a common life threaten disease, however, the diagnostic efficiency is still not optimal. Developing a novel method to improve diagnosis is necessary. The aim of this project

17 was to formulate a material that can bind to GPC3 of HCC for targeted enhanced ultrasound. 
18 Methods. A material of sulfur hexafluoride $\left(\mathrm{SF}_{6}\right)$ filled liposome microbubbles and conjugated

19 with synthesized peptide (LSPMbs) was prepared and assessed in vitro and vivo. Liposome

20 microbubbles were made of DPPC, DPPG, DSPE-PEG2000, and $\mathrm{SF}_{6}$, using thin film method to

21 form shell, followed filling $\mathrm{SF}_{6}$, and conjugating peptide. A carbodiimide method was used for

22 covalent conjugation of peptide to LSMbs.

23 Results. The prepared LSPMbs appeared round shaped, with size of $380.9 \pm 176.5 \mathrm{~nm}$, and Zeta potential of $-51.4 \pm 10.4 \mathrm{mV}$. LSPMbs showed high affinity to Huh-7 cells in vitro, presented good enhanced ultrasound effects, did not show cytotoxicity, and did not exhibit targeted fluorescence and enhanced ultrasound in animal xenograft tumors.

Conclusion. Extravascular contrast-enhanced ultrasound targeted GPC3 on HCC may not be realized, and the reason may be that targeted contrast agents of microbubbles are hard to access and accumulate in the tumor stroma and matrix. 


\section{Introduction}

Hepatocellular carcinoma (HCC) is a common primary malignant neoplasm derived from hepatocytes, especially in some Asia-Pacific regions, where the underline diseases of hepatitis B virus infection and relevant diseases are in high prevalence [1]. At present, the diagnosis of small and atypical HCC is still challenging [2]. Healthcare professionals and scientists have been searching new methods to improve diagnosis efficiency. Targeted imaging has been a heat topic and interest of researchers in recent decades, and is expected to be an ideal non-invasive imaging method [3-5]. Although the lack of a basement membrane and smooth muscle and the expansion of the intercellular space in cancer vasculature result in a maximum pore size of approximately 380-780 nm, which exhibits leaky and/or defective blood vessels, microbubbles (Mbs) with diameter more than $1000 \mathrm{~nm}$ cannot migrate from the tumor vasculature to the cellular target site to exert the desired diagnostic effect [3]. Therefore, the development of nanoscale targeted ultrasound contrast agents (UCAs), which may permeate through the tumor vasculature gap and bind to tumor cells, with extravascular imaging function, is required. On HCC cellular membrane, there is a high expression of Glypican-3 (GPC3) protein, which can be used for a target for molecular imaging $[4,5]$. However, because of the antigenicity and larger size of GPC3, if it is used for the ligand of a targeted material, the material may not produce desired effect in the vivo. On this condition, if a small size peptide without antigenicity but possessing similar targeting ability, it may be used for the fabrication of a targeted contrast material. We hypothesized that a new material may be fabricated, with function of targeted contrast-enhanced ultrasound (CEU) imaging. Based on previous studies, we established a sort of liposome microbubbles and conjugated with a synthesized peptide targeting GPC3 of the HCC, with liposome as shell and sulfur hexafluoride gas $\left(\mathrm{SF}_{6}\right)$ as the core $[4,5]$.

\section{Materials \& Methods}

\section{Ethics statement}

All the experimental procedures in this study were in compliance with the National Institutes of Health guidelines and were approved by the Institutional Animal Care and Use Committee of Hainan Medical University (2018-02-27). 


\section{Preparation of Liposomes and Liposome Microbubbles}

Sulfur hexafluoride gas $\left(\mathrm{SF}_{6}\right)$ filled liposome microbubbles and conjugated with synthesized peptide (LSPMbs), $\mathrm{SF}_{6}$ filled liposome microbubbles and conjugated with GPC3 antibody (LSGMbs), $\mathrm{SF}_{6}$ filled liposome microbubbles and conjugated with synthesized peptide, and liposomes (LS) were prepared and assessed in the public scientific research center of Hainan medical university. To develop a GPC3 targeted liposome microbubbles, materials and formula were chosen and optimized, and the optimum formula was selected through orthogonal design test according to the enhanced ultrasound effect. 1,2-dipalmitoyl-sn-glycero-3-phosphocholine (DPPC), 1,2-Dipalmitoyl-sn-glycero-3-phospho-sn-1-glycerol (DPPG), 1,2-distearoyl-snglycero-3-phosphoethanolamine-N-[carboxy(polyethylene glycol)-2000] (DSPE-PEG2000), GPC3, artificial synthesized peptide of DHLASLWWGTEL and SF $_{6}$ were adopted and fabricated using following protocols. $12 \mathrm{mg}$ DPPC was dissolved in $3.0 \mathrm{~mL}$ chloroform, followed adding $1.5 \mathrm{~mL}$ methanol and $0.5 \mathrm{~mL}$ deionized water to form a mixture, next dissolved $23 \mathrm{mg}$ DPPG and 11mg DSPE-PEG2000 in the mixture. The solvent was removed by rotary evaporation and vacuum using a Vacuum Rotary Evaporator (Xian Depai17 Biotechnology Co.,

Ltd. Xian, China), and the mixture formed a thin film on the wall of the container. $20 \mathrm{~mL}$ deionized water was added to the container to harvest and suspend the lipid mixture, next it was transferred to a tube to undergo magnetic stirring at $65^{\circ} \mathrm{C}$ for $20 \mathrm{~min}$, followed by probesonication (Vibracell ${ }^{\mathrm{TM}}$, VCX130PB/VCX130 Sonics, Sonics and Materials, Inc., Newtown, USA) with a frequency of $40 \mathrm{kHz}$ under $35^{\circ} \mathrm{Cfor} 5 \mathrm{~min}$. A mechanical blender (Ultra-turrax T25, Janke \& Kunkel, IKA-Labortechnik, Staufen, Germany) was used for liposome microbubbles 
preparation. The above processed lipid dispersion was put into a $50 \mathrm{~mL}$ Falcon tube, the air above the aqueous dispersion in the tube was replaced with $\mathrm{SF}_{6}$ gas, and the tube was sealed with parafilm. The temperature of lipid dispersion was heated to $30^{\circ} \mathrm{C}$, the homogenizer was operated to create high shear mixing (15000 rpm, $5 \mathrm{~min})$ to form microbubbles. The mixture was centrifuged at $12000 \mathrm{rpm}$ at $4^{\circ} \mathrm{C}$ for $5 \mathrm{~min}$, washed with deionized water, three times. $15 \%(\mathrm{w} / \mathrm{v})$ sucrose solution was added to the mixture in a 5:1 volume ratio (mixture: sucrose), small glass vial of $4 \mathrm{~mL}$ volume was used for the loading, each with $2 \mathrm{~mL}$ mixture. The air in the vials was replaced with $\mathrm{SF}_{6}$ gas before lyophilized in a $-85^{\circ} \mathrm{C}$ lyophilizer (SP Scientific, VirTis, USA). After $24 \mathrm{~h}$ completely freeze-drying, vials were refilled with $\mathrm{SF}_{6}$ gas and sealed, stored at $4^{\circ} \mathrm{C}$. Pure liposomes (LS) were prepared using the above protocols without filling $\mathrm{SF}_{6}$ gas.

\section{Preparation of GPC3 Targeted Liposome Microbubbles}

Firstly, after liposome microbubbles (LSMbs) were prepared using the protocols above, but did not add sucrose solution and lyophilize. Next, a carbodiimide method was used for covalent conjugation of the synthesized peptide to the free carboxyl groups on the surface of LSMbs. The prepared LSMbs were resuspended in MES buffer $(0.1 \mathrm{~mol} / \mathrm{L}, \mathrm{pH} 6.0)$, and an adequate amount of EDC/NHS [1-ethyl-3(3-dimethylaminopropyl) carbodiimide (EDC): N-hydroxy succinimide $(\mathrm{NHS})=1: 4$, Sigma-Aldrich Chemical Co., Inc, USA] were added into the suspension. The mixture suspension was oscillated and incubated for $2 \mathrm{~h}$ at room temperature $\left(25^{\circ} \mathrm{C}\right)$. The remaining EDC/NHS was removed by three-time centrifugation at $12000 \mathrm{rpm}$ using MES (pH 6.0), 5 min each time. The precipitate was dispersed into MES buffer $(0.1 \mathrm{~mol} / \mathrm{L}, \mathrm{pH} 8.0)$, and an adequate amount of synthesized peptide was added and incubated with stirring for $2 \mathrm{~h}$ at room 
110

111

112

temperature. The peptide was compounded by GL Biochem (Shanghai) Ltd. (Shanghai, China)

in accordance to a 12-mer peptide with the sequence of DHLASLWWGTEL reported from

previous study that it can target GPC3 of HepG2 [4] [ DOI: 10.1021/acs.bioconjchem.6b00030].

The mixture was centrifuged at $12000 \mathrm{rpm}$ at $4^{\circ} \mathrm{C}$ for $5 \mathrm{~min}$, washed by deionized water, three

times. Next, 15\% (w/v) sucrose solution was added to the mixture in a 5:1 volume ratio (mixture:

sucrose), small glass vial of $4 \mathrm{~mL}$ volume was used for the loading, each with $2 \mathrm{~mL}$ mixture. The

air in the vials was replaced with $\mathrm{SF}_{6}$ gas before lyophilized in a $-85^{\circ} \mathrm{C}$ lyophilizer (SP Scientific,

VirTis, USA). After $24 \mathrm{~h}$ completely freeze-drying, vials were refilled with $\mathrm{SF}_{6}$ gas and sealed,

stored at $4^{\circ} \mathrm{C}$. Liposome microbubbles (LSMbs), and GPC3 antibody (BM1846; Wuhan Boster

Biological Technology, Ltd., Wuhan, China) conjugated liposome microbubbles (LSGMbs) were prepared in the similar protocol above.

\section{Characterization of LSPMbs}

\section{Transmission Electron Microscopy Evaluation}

The LSPMbs were observed using transmission electron microscope (TEM) for particle size and shape assessment. LSPMbs were suspended with deionized water (1:50), and one drop of the suspension was dropped onto a carbon-coated copper grid. After drying and adhesion in $25^{\circ} \mathrm{C}$, samples were negatively stained by sodium phosphotungstate solution $(2 \%, \mathrm{w} / \mathrm{w})$ and analyzed with a 120-kV TEM (TEM; JEM 2100, JEOL, Tokyo, Japan). Suspensions of samples with different concentration were dropped on coverslips and observed under light microscopy.

\section{LSPMbs Size and Zeta Potential Measurements}


130 LSPMbs size and Zeta potential of each sample was measured using a Zetasizer Nano S90

131 (Malvern Instruments Ltd., Malvern, UK) by Laser Doppler Anemometry (LDA) using

132 electropheoretic light scattering at $25^{\circ} \mathrm{C}$. An adequate amount of LSPMbs

133 was suspended and diluted to enable the microbubbles concentration was maintained to ensure

134 that multiple scattering and microbubble-microbubble interactions were negligible. The

135 microbubble size and zeta potential of each sample were measured three times, and the mean

136 value was taken as the final microbubble size and zeta potential. LSGMbs were assessed in the

137 same methods.

138

139

140

141

142

143

\section{Assessment of Biocompatibility of LSPMbs}

\section{MTT Assay for Cytotoxicity}

RAW 264.7 cells [Cell bank of the Chinese academy of sciences (Shanghai, China)] in exponential phase of growth were taken, transferred $200 \mu \mathrm{L}$ to each well of a 96-well plate, and adjust the cell density to 5000/well. The cells were cultured with DMEM (Wuhan Boster Biological Technology, Ltd., Wuhan, China), 5\% fetal bovine serum (FBS) (Gibco, Australia), and $1 \%$ penicillin-streptomycin at $37{ }^{\circ} \mathrm{C}$ in $5 \% \mathrm{CO}_{2}$ atmosphere for $24 \mathrm{~h}$, when the well were fully covered, added different concentration gradients LSPMbs $(2 \mathrm{mg} / \mathrm{mL}, 5 \mathrm{mg} / \mathrm{mL}, 10 \mathrm{mg} / \mathrm{mL}$, $15 \mathrm{mg} / \mathrm{mL}$, and $20 \mathrm{mg} / \mathrm{mL}$ ) prepared using with DMEM and 5\% FBS to different wells, $200 \mu \mathrm{L}$ per well, then continuing incubated for $24 \mathrm{~h}$. LSPMbs were burst using ultrasound at the experiment. Next, $10 \mu \mathrm{L}$ 0.5\% 3-(4,5-dimethylthiazol-2-yl)-2,5-diphenyl-2H-tetrazolium bromide (MTT) solution was added to each well, and continuing incubated for $24 \mathrm{~h}, 48 \mathrm{~h}$ and $72 \mathrm{~h}$ in the dark place, respectively. The incubation was ended at $24 \mathrm{~h}, 48 \mathrm{~h}$ and $72 \mathrm{~h}$, respectively, and 
151 the solution in the wells were carefully absorbed out and discarded. Next, $200 \mu \mathrm{L}$ dimethyl

152 sulfoxide was added to each well, and oscillated at a frequency of 20 times per minute for $10 \mathrm{~min}$

153 to fully dissolve the crystal. Optical density $(O D)$ is measured at the $570 \mathrm{~nm}$ wavelength by

154 using a spectrophotometric microplate reader (Bio-Tek ELX-800, Winooski, VT, USA).

155 Controls were established and processed using the similar protocols.

156

157

158

159

160

161

162

163

164

165

\section{Evaluation of Enhanced Ultrasound Imaging of LSPMbs in Vitro}

To assess the contrast-enhanced effect of LSPMbs, LSPMbs were suspended with deionized water and diluted into various concentrations $(1.6,0.8$, and $0.4 \mathrm{mg} / \mathrm{mL})$ and placed in different plastic tubes for ultrasound evaluation. LSPMbs suspension filled tubes were fixed in a water container, and their CEU effect was assessed using GE Logiq E9 ultrasound system (GE Healthcare, Milwaukee, WI, USA), using a ML6-15-D linear transducer with a frequency of 4$15 \mathrm{MHz}$. During the ultrasound performance, the frequency of the transducer was set to $12 \mathrm{MHz}$, the depth and focus were adjusted to optimize imaging, and the model was shifted to contrast imaging, using the default parameter (MI 0.1). Before the ultrasound scanning, the tubes were agitated slightly. Controls of commercial ultrasound contrast agent SonoVue (Shanghai Bracco Sine Pharmaceutical Corp. Ltd., Shanghai, China), degas deionized water, and air were established, and these were assessed using the same protocols above.

Enhancement ultrasound imaging effects of LSPMbs, LSGMbs, SonoVue, LS, degas deionized water, and air were determined using Photoshop software (Adobe Photoshop CS3, Adobe Systems Inc, CA, USA). To analyze ultrasound images, observers opened the image in 
171 Photoshop, activated menus of "Window, Information, and Histogram" consecutively, selected

172 "rectangle, and statistics display" tools, set the same region of interest to the tube, parameters

173 yielded automatically, measured three times in each image, and adopted the mean value of scales

174 (arbitrary units) as the result of a single image.

175

176

177

178

\section{Assessment of Affinity of LSPMbs to the Liver Cancer Cells}

Fluorescence experiment was used for the assessment of affinity of LSPMbs to the liver cancer cells. A tiny amount of 1,1'-dioctadecyl-3,3,3',3'-tetramethylindocarbocyanine perchlorate (DiI)

(Yeasen Biotech Co., Ltd., Shanghai, China) was added into 5mL of $16 \mathrm{mg}$ LSPMbs suspension to form DiI labelled LSPMbs suspension. Huh-7 cells [Cell bank of the Chinese academy of sciences (Shanghai, China)] were seeded in a six-well plate with one glass slip placed in each well at a concentration of $3 \times 10^{4}$ cells/well. Next day, the Huh-7 cells were fixed by $90 \%$ cold ethanol for $20 \mathrm{~min}$ and blocked by $10 \%$ bovine serum albumin (BSA) at $37^{\circ} \mathrm{C}$ for $1 \mathrm{~h}$ and subsequently incubated with 4 drops of DiI labelled LSPMbs suspension for $3 \mathrm{~h}$ in dark place.

Next, the wells and slips were washed with PBS three times, and the cells on the slips were mounted with 4', 6-diamidino-2-phenylindole (DAPI, Wuhan Boster Biological Technology, Ltd., China) for nuclei visualization and detected using a laser confocal microscope (Fluoview FV 10001000, Olympus, Japan). Images of bright, DAPI staining, DiI staining, and merged were obtained. LSGMbs were assessed in the same protocols for control. To confirm the specificity of binding of DiI labelled LSPMbs to GPC3 in the Huh-7 cells, $0.1 \mathrm{~mL}$ synthesized peptide 
$190(10 \mu \mathrm{g} / \mathrm{mL})$ was applied before adding DiI labelled LSPMbs as a blocking control, and the other

191 protocols were the same as the above.

192

193

194

195

196

\section{Assessment of LSPMbs in Vivo}

All animal experimental procedures were approved by the Our University Association for Accreditation of Laboratory Animal Care. Animals of female BALB/C mice were used for the evaluation of targeting ability and contrast-enhanced effect. Twenty Huh-7 cell xenograft tumor models of female BALB/C mice were established, and the Huh-7 cell line was acquired from the cell bank of the Chinese academy of sciences (Shanghai, China). All animals expect four mice were sacrificed by euthanasia using isoflurane after fluorescent imaging were sacrificed using carbon dioxide in a closed box at the end of the animal experiments. The criteria of animal death are that the mice were in collapse state, losing muscular tension, no breath, no heart beating, and the skin color became gray.

\section{Enhanced Ultrasound Imaging Assessment of LSPMbs}

Fifteen of the mice were used for the targeted CEU experiments in five groups, with each group of three mice. The CEU effect was assessed using GE Logiq E9 ultrasound system (as has addressed in the previous section). During the ultrasound scanning, the frequency of the transducer was set to $12 \mathrm{MHz}$, the depth and focus were adjusted to optimize imaging, and the model was shifted to contrast imaging, using the default parameter (MI 0.1). The shape of the xenograft tumors was ovoid, and the longitudinal diameter of the tumors of 24 mice was $10.4 \pm 0.53 \mathrm{~mm}$ in the fifth week after cells seeded. Control experiments were conducted in four 
210 groups using LSGMbs, LSPMbs (GPC3 antibody or synthesized peptide blocked previously),

211 SonoVue (a commercial ultrasound contrast-enhanced agent), and LS, respectively. LSPMbs

212 suspension was prepared using 14mg LSPMbs and $4 \mathrm{~mL} 0.9 \%$ sodium chloride solution, agitated

213 slightly before injection. The animals with Huh-7 xenograft tumor in five groups were

214 intravenously injected suspension of LSPMbs, LSGMbs, LSPMbs (injected 0.1mL synthesized

215 peptide or $0.1 \mathrm{~mL}$ dilated GPC3 antibody for blocking in 3 minutes ahead), SonoVue (15mg

216 SonoVue in $4 \mathrm{~mL}$ 0.9\% sodium chloride solution), and LS (14mg LS and $4 \mathrm{~mL} 0.9 \%$ sodium

217 chloride solution, agitated slightly before injection), respectively, each with the volume of

$0.2 \mathrm{~mL}$. The contrast imaging time was counted since the bolus intravenous injection of $0.2 \mathrm{~mL}$

219

220

221

222

223

224

225

226

227

228

229

LSPMbs suspension via the mouse tail vein. The images were saved in the ultrasound system and exported for study late.

Enhancement ultrasound imaging effects of LSPMbs, LSGMbs after synthesized peptide or GPC3 antibody blocking, SonoVue, LS were determined using Photoshop software, and the methods had been addressed in the previous section.

\section{Fluorescent Imaging Assessment of LSPMbs}

Ten mice with xenograft tumors of Huh-7 cells allotted to two groups of five each were used to conduct Cy 5.5 fluorescence experiment to test the specificity and affinity of the peptide in LSPMbs to GPC3 of the liver cancer. An IVIS Lumina image system (Xenogen) (IVIS ${ }^{\circledR}$ Lumina XR) (Caliper life sciences) was used for the evaluation. During the fluorescence imaging, mice were under gas anesthesia with oxygen and isoflurane (Jinan Shengqi pharmaceutical Co, Ltd., 
230 Jinan, China). 0.2mL Cy5.5 labelled LSPMbs suspension was intravenously injected into six

231 mice with Huh-7 xenograft tumors via the tail veins, images were acquired at one minute, six

232 hours, and 24 hours. Cy5.5 labelled LSPMbs suspension was prepared using 14mg Cy5.5

233 labelled LSPMbs and $4 \mathrm{~mL} 0.9 \%$ sodium chloride solution, agitated slightly before injection.

234 The control experiments were conducted in five mice with Huh-7 xenograft tumors, with the

235 same methods after injection of $0.1 \mathrm{~mL}$ synthesized peptide $(10 \mu \mathrm{g} / \mathrm{mL})$ for blocking in three

236 minutes. Four mice were sacrificed by euthanasia using $3 \mathrm{~m} \mathrm{~L}$ isoflurane in a closed small box.

237 The tumor, liver, heart, lung, kidney, and spleen of the mice were isolated for fluorescent

238 imaging assessment at 24hours.

239 Statistical analysis

240 Quantitative data are presented as mean \pm SD (standard deviation), and qualitative data are

241 presented as percentile. Statistical significance of differences between groups of quantitative

242 variables were analyzed using paired-sample $t$ tests or univariate analysis of variance, and

243 qualitative variables were analyzed using Chi-square test. All statistical analyses were performed

244 using SPSS software (Version 20; IBM, Armonk, NY, USA). $P<0.05$ was considered

245 significant.

246 Results

247 Characterization of LSPMbs

248 The LSPMbs appeared round shaped on a sectional view, with different size, without

249 aggregation, identified by transmission electron microscopy (Figure 1). 
250 Size and Zeta potential of LSPMbs were $380.9 \pm 176.5 \mathrm{~nm}$ and $-51.4 \pm 10.4 \mathrm{mV}$, respectively. The

251 determination results showed "Good" (Figures 2 and 3).

252 Assessment of Biocompatibility of LSPMbs

253 MTT Assay for Cytotoxicity

254 The cell index had no significant differences among different LSPMbs concentrations at different

255 time (all $P>0.05$ ), indicating that LSPMbs did not cause significant toxic effect on RAW 264.7

256 cells. As shown on Figure 4.

257

258

259

260

261

262

263

264

265

266

267

268

269

\section{Evaluation of Enhanced Ultrasound Imaging of LSPMbs in Vitro}

LSPMbs and SonoVue suspension with different concentrations presented different enhancement effects, they presented almost the same enhancement effect at the same concentration (Figures 5

A and $\mathrm{D}$ were obtained from $1.6 \mathrm{mg} / \mathrm{mL}$, figures $5 \mathrm{~B}$ and $\mathrm{E}$ was obtained from $0.8 \mathrm{mg} / \mathrm{mL}$, and

figures $5 \mathrm{C}$ and $\mathrm{F}$ was obtained from $0.4 \mathrm{mg} / \mathrm{mL})$. At higher concentration $(1.6 \mathrm{mg} / \mathrm{mL})$, they all

presented homogeneous hyperechogenicity with marked attenuation (Figures 5A and D), and the echogenicities became weaker when the concentrations decreased (Figures 5B and E, and figures

5C and F), and they all much stronger than control of degas deionized water, which presented

homogeneous anechogenicity (Figure 5G). Control of air presented strong echogenicity at the

interface of the tube, and the distal field presented marked attenuation (Figure 5H), which was

substantial different from those obtained from LSPMbs and SonoVue suspension.

Comparisons of measurements of enhanced ultrasound imaging between different

concentrations and agents were as follow: figure A vs. $\mathrm{D}, P=0.321$; figure $\mathrm{B}$ vs. $\mathrm{E}, P=0.472$; 
270 figure $\mathrm{C}$ vs. F, $P=0.428$; and figure A vs. G, A vs. H, B vs. H, G vs. H, A vs. B, A vs. C, B vs. C,

271

272

273

274

275

276

277

278

279

280

281

282

283

284

285

286

287

288

289

290

D vs. E, and D vs. F, all $P<0.05$.

\section{Assessment of Affinity of LSPMbs to the Liver Cancer Cells}

The fluorescence on the cellular membrane of Huh-7 cells was intensive (Figure 6), indicating that there was high GPC3 expression. Cells and DiI labled LSPMbs and controls obtained from light microscope (Figure 7 A, E and I). Cell nucleus of Huh-7 cells presented blue after DAPI staining and being incubated with DiI labled LSPMbs (Figure 7 B, F and J). LSPMbs with DiI staining combined with the membrane of Huh-7 cells presented red color fluorescence on image (Figure $7 \mathrm{C}$ and D); LSGMbs presented a very similar appearance (Figure $7 \mathrm{G}$ and $\mathrm{H}$ ); and LSPMbs with DiI staining after synthesized peptide blocking previously did not show red color fluorescence on the membrane of Huh-7 cells, indicating that LSPMbs had not combined with the membrane of Huh-7 cells (Figure $7 \mathrm{~K}$ and L).

\section{Assessment of LSPMbs in Vivo}

\section{Enhanced Ultrasound Imaging Assessment of LSPMbs}

All xenograft tumors in the mice of the five groups presented a complex of isoechogenicity, hypoechogenicity and anechogenicity (Figures A, E, I, M and Q). Of the study group, after administration of LSPMbs suspension, Huh-7 xenograft tumor presented hyper-enhancement in periphery and hypo-enhancement in center (necrosis) at two seconds (Figure $8 \mathrm{~B}$ ); the tumor enhancement lasted over 20 seconds with little change; at 60 seconds, the tumor still presented hyper-enhancement in periphery and hypo-enhancement in center (necrosis) (Figure $8 \mathrm{C}$ ); at 10 minutes, the tumor presented iso-enhancement with central hypo-enhancement (necrosis) (Figure 
2918 D). Of the four control groups, the mice injected with LSGMbs, LSPMbs (blocked with GPC3

292 antibody or synthesized peptide previously), and SonoVue, respectively, presented similar

293 enhancement patterns and sustain time as those in Huh-7 xenograft tumors (Figures 8 F, G and

$294 \mathrm{H}$, Figures $8 \mathrm{~J}, \mathrm{~K}$ and L, and Figures $8 \mathrm{~N}, \mathrm{O}$ and P), and there was no significant difference; the

295 xenograft tumors presented similar enhancement patterns and sustaining time; of the mice in the

296 group injected with LS suspension, the xenograft tumors did not present enhancement at 2, 20,

29760 seconds, and 10 minutes (Figures $8 \mathrm{R}, \mathrm{S}$ and T).

298 Comparisons of enhanced ultrasound imaging among different agents at times of two seconds, 20

299 seconds, and 10 minutes, scales of images ( Figures B, C, and D of LSPMbs, figures J, K, and L

300 of LSPMbs after synthesized peptide or GPC3 blocking, figures F, G, and H of LSGMbs, and

301 figures $\mathrm{N}, \mathrm{O}$, and $\mathrm{P}$ of SonoVue) all had no significant difference (all $P>0.05$ ), and there were

302 significant difference between the above scales of images and scales of LS images (all $P<0.001$ ).

303 These indicate that LSPMbs has good capability in CEUS imaging, but the experiments of it did

304 not show targeted imaging in vivo.

\section{Fluorescent Imaging Assessment of LSPMbs}

306 The fluorescent signal could be visualized all over the body soon after the administration of

307 Cy5.5 labelled LSPMbs suspension. Images acquired at one minute (Figure 9A), six hours

308 (Figure 9B) after the initial fluorescent imaging, the fluorescent signal intensity in the tumor area

309 has no significant difference from other areas of the body expect the liver and spleen. The

310 fluorescent signal intensity in the liver and spleen area of the mice was marked stronger than

311 other areas, and the fluorescent signal intensity was similar in three times. The fluorescent signal 
312 could not be visualized in the mice 24 hours after injection (Figure 9C). Experiment carried out

313 in the mice blocked previously by injection of GPC3 antibody or synthesized peptide presented

314 the same fluorescent imaging characteristics as those in the mice with Huh-7 xenograft tumors

315 (Figures 9D, E and F). 24 hours after intravenous injection of Cy5.5 labelled LSPMbs, four mice

316 with Huh-7 cell xenograft tumors of two in each groups were sacrificed, the tumors and visceral

317 organs were assessed, there were fluorescence in the lungs and liver, and no fluorescence in the

318 tumor and the heart, spleen and kidneys (Figure 10). These indicate that the Cy5.5 labelled

319 LSPMbs did not selective accumulated in the xenograft tumor, and the LSPMbs did not present

320 detectable targeting ability to the tumor. The reason that the fluorescent signal intensity in the

321 liver and spleen was higher than other areas is believed that the liver and spleen have abundant

322 capillaries and macrophage cells, the macrophage cells can engulf the liposomes, so liposomes in

323 these regions are richer than other regions. The more Cy5.5 labelled LSPMbs aggregated, the

324 stronger the fluorescent signal intensity.

325 Discussion

326

327

328

329

330

331

LSPMbs presented similar enhancement effect at the same concentration as that the SonoVue performed in vitro and vivo, indicating that the LSPMbs has good capability of enhancement imaging. Optical imaging in vivo using fluorescence and bioluminescence has high sensitivity and resolution [6]. The near infrared dye Cy5.5 allows a fluorescent imaging of deep tissue in rodent animals with low background, providing a possibility of evaluation of the molecular imaging agents. In this study, LSPMbs were labelled with Cy5.5 for targeting GPC3 imaging 
332 evaluation, the results showed that they had not presented aggregated fluorescence imaging, 333 indicating that LSPMbs were not targeting retained in the tumor. LSPMbs did not present 334 targeted imaging both at ultrasound imaging and optical imaging. extravascular tissue, common UCAs are confined to the blood pool when administered intravenously, which are consist of microbubbles in suspension which strongly interplay with the ultrasound beam and are readily detectable by ultrasound imaging systems [7]. Molecularly targeted UCAs are created by conjugating the microbubble shell with a peptide, antibody, or other ligand designed to target an endothelial biomarker associated with tumor angiogenesis or inflammation. These microbubbles then accumulate in the microvasculature at target sites where they can be imaged [7]. growth factor receptor 2 (VEGFR2) based targeted UCAs [8]. These targeted UCAs do not need to extravasate the blood vessels. The VEGFR2 based targeted UCAs can contact and combine with the VEGFR2 on the blood vessels when they enter and flow through the tumor's vessels, forming focal contrast agent accumulation, and can display focal enhanced imaging at ultrasound scanning. However, our preparation of LSPMbs targeting cellular membranous receptor of the HCC confronts substantial challenge for targeted imaging. Sizes of LSPMbs are $380.9 \pm 176.5$ $\mathrm{nm}$, which can extravasate the fissure of blood vessel wall of tumor if there are no other impeding factors. But the experimental results did not gain the desired goal. The reasons may be 
352 the following factors. The previous study showed that the gap between the epithelia of cancer

353 blood vessel is large enough (380-780 nm) to allow nanoscale materials passing, but the blood

354 vessels contact the cells of tumor and interstitial closely, elevated interstitial fluid pressure in the

tumor could restrict convective flow and antibody extravasation, except in large necrosis and

hypoxia areas $[9,10]$. Similarly, the targeted LSPMbs needs overcome interface pressure gradient

and get enough space to access and bind to the cancer cells of tumor. How the LSPMbs

penetrate the blood vessels of tumor, distribute in the tumor and uptake by the cells are difficult

to understand or predict [11]. A study by Opie showed that the osmotic parameters of tumors

(hepatoma, etc) are much lower than that of normal tissues [12]. In this circumstance, the

suspension of LSPMbs is harder to be absorbed into tissue by osmotic force of tumor. At tumor

sites, the disorganized tumor vascular network, extensively distributed stromal cells (e.g., tumor-

associated macrophage, cancer-associated fibroblasts, etc.) and the dense physical barriers of

extracellular matrix comprise of the abominable obstacles hampering nanoparticles transport in a

tumor. [13] The electron microscopy results on a study have confirmed that the opening in tumor

extracellular matrix barriers surrounding the cancer cells is generally less than $40 \mathrm{~nm}$.[14] On

this condition, LSPMbs of $380.9 \pm 176.5 \mathrm{~nm}$ are impossible to pass the extracellular matrix

opening to access to the cancer cells. A recent study revealed that only $0.7 \%$ of systemically

administered nanoparticles can reach the tumor sites and less than 14 out of 1 million $(0.0014 \%$

injected dose) of them are accessed by cancer cells, and that only 2 out of 100 cancer cells

interacted with the nanoparticles.[15] Therefore, if the number and volume of the targeted

LSPMbs entering the tumor extravascular part are not enough, it will be impossible to yield 
373 visible enhanced imaging effect.

374 Many researches on extravascular targeted contrast-enhanced ultrasound have been reported

375 in literature, but only a few of them validate in vivo of animals. Mai et al reported that a

376 chitosan-vitamin C lipid system had been fabricated and had achieved tumor-selective enhanced

377 ultrasound imaging in a mouse tumor model, but they confirmed only that the fluorescence

378 accumulated highly at the tumor site, other than the nanobubbles [16]. Another extravascular

379 targeted nanobubbles fabricated by Gao et al remains to be further verification because of the

380 preliminary results and substantial limitations [17]. Theoretically, if microbubbles enter the

381 tumor interstitials, some liquid solution also enters. There must be enough extravascular space in

382 the tumor to contain and distribute them, only in this condition can the microbubbles in the

383 oscillations of ultrasonic compression and expansion wave generate stronger backscattered

384 acoustic signal and second harmonics for enhanced ultrasound imaging. If many microbubbles

385 are compacted together, their size will be big, and which will generate little backscattered

386 acoustic signal and second harmonics [18].

387 Our experimental results, together with earlier published reports by others[13-15], strongly

388 suggested that to develop a targeted material for extravascular contrast-enhanced ultrasound

389 imaging, cutting-edge precisive experiments should be conducted firstly to ascertain that the

390 material can penetrate the blood vessel and wade through the cellular matrix and stroma to

391 access and bind to the target cells, and the accommodation space for the materials is adequate. In

392 future, the development of materials for extravascular contrast-enhanced ultrasound imaging 
393

394

395

396

397

may be emphasis that using specific materials such as cell-penetrating peptides, a disulfidebridged cyclic RGD peptide, named iRGD (internalizing RGD, c(CRGDK/RGPD/EC)), which is a tumor-homing peptide that can bind to avb3 integrin with high affinity and specificity to construct the targeted material. A material integrated iRGD peptide may increase penetration of the blood vessels and matrix, and facilitate accumulation and increase the probability of enhanced imaging $[19,20]$. Augmentation of enhanced permeability and retention effect of targeted material through using NO-releasing agent such as nitroglycerin or angiotensinconverting enzyme inhibitors, and albumin-protein interactions using S -nitrosated human serum albumin dimer, etc, may increase targeted material accumulation and the probability of enhanced imaging [21,22].

\section{Conclusions}

Collectively, a new material of LSPMbs has been prepared, which has good effect of enhanced ultrasound imaging, but it did not exhibit targeted imaging effect in vivo of animal experiments. The causes may be that the volume of LSPMbs pass the tumor blood vessels and enter the tumor parenchyma was very limited, and the LSPMbs cannot pass the fissures of extracellular stroma and matrix surrounding the cancer cells to access and bind to the cancer cells. Therefore, a potential target of GPC3 on hepatocellular carcinoma for extravascular targeted imaging may not be realized in contrast-enhanced ultrasound. The future research should focus on that whether a candidate targeted material for extravascular contrast-enhanced ultrasound imaging can penetrate the blood vessel and wade through the cellular matrix and 
413 stroma to access and bind to the target cells, and whether the accommodation space for the

414 materials is adequate.

\section{Acknowledgements}

416 None.

\section{References}

418 1. Zhu RX, Seto WK, Lai CL, Yuen MF. 2016. Epidemiology of hepatocellular

419 carcinoma in the Asia-Pacific region. Gut Liver 10:332-339

420 2. Cassinotto C, Aubé C, Dohan A. 2017. Diagnosis of hepatocellular carcinoma: An update on 421 international guidelines. Diagn Interv Imaging 98:379-391

422 3. Ofuji K, Saito K, Yoshikawa T, Nakatsura T. 2014. Critical analysis of the potential of 423 targeting GPC3 in hepatocellular carcinoma. J Hepatocell Carcinoma 1:35-42

424 4. Zhu D, Qin Y, Wang J, Zhang L, Zou S, Zhu X, Zhu L. 2016. Novel Glypican-3-binding 425 Peptide for in vivo Hepatocellular Carcinoma (HCC) Fluorescent Imaging. Bioconjug Chem $426 \quad 27: 831-839$

5. Wu Size. 2017. Formation and characterization of nanobubbles conjugating peptide targeting GPC3 of hepatocellular carcinoma. J Nanosci Nanotechno17: 4542-4548

6. Imamura T, Saitou T, Kawakami R. 2018. In vivo optical imaging of cancer cell function and tumor microenvironment. Cancer Sci 109:912-918 
431 7. Chong WK, Papadopoulou V, Dayton PA. 2018. Imaging with ultrasound contrast agents:

432 current status and future. Abdom Radiol (NY) 43:762-772

433 8. Willmann JK, Bonomo L, Testa AC, Rinaldi P, Rindi G, Valluru KS, Petrone G, Martini M, 434 Lutz. 2017. Ultrasound Molecular Imaging With BR55 in Patients With Breast and Ovarian 435 Lesions: First-in-Human Results. J Clin Oncol 35:2133-2140 9. Wang S, Herbst EB, Mauldin FW Jr, Diakova GB, Klibanov AL, Hossack JA. 2016. Ultralow-dose Ultrasound Molecular Imaging for the Detection of Angiogenesis in a Mouse Murine Tumor Model: How Little Can We See? Invest Radiol 51: 758-766

10. Thurber GM, Schmidt MM, Wittrup KD. 2008. Factors determining antibody distribution in tumors. Trends Pharmacol Sci 29:57-61

11. Jain RK. Transport of molecules, particles, and cells in solid tumors. 1999. Annu Rev

Biomed Eng 1:241-263

12. Opie E L. 1949.The movement of water in tumor tissue removed from the body. J Exp Med

89: $209-222$

13. Zhang Z, Wang H, Tan T, Li J, Wang Z, Li Y. 2018. Rational design of nanoparticles with deep tumor penetration for effective treatment of tumor metastasis. Adv Funct Mater 28:

$447 \quad 1801840$

448 14. Ng K K, Lovell J F, Zheng G. 2011. Lipoprotein-inspired nanoparticles for cancer theranostics. Acc Chem Res 44: 1105-1113 
450 15. Dai Q, Wilhelm S, Ding D, Syed A M, Sindhwani S, Zhang Y. 2018. Quantifying the

451 ligand-coated nanoparticle delivery to cancer cells in solid tumors. ACS Nano 12: 8423-8435

452 16. Mai L, Yao A, Li J, Wei Q, Yuchi M, He X, Ding M, Zhou Q. 2013. Cyanine 5.5 conjugated

453 nanobubbles as a tumor selective contrast agent for dual ultrasound-fluorescence imaging in a

454 mouse model. PLoS One 8: e61224

455 17. Gao Y, Hernandez C, Yuan HX, Lilly J, Kota P, Zhou H, Wu H, Exner AA. 2017.

456 Ultrasound molecular imaging of ovarian cancer with CA-125 targeted nanobubble contrast

457 agents. Nanomedicine 13: 2159-2168

458 18. Sanchez, MV, Varadarajulu, S, Napoleon, B. 2009. EUS contrast agents: what is available,

459 how do they work, and are they effective? Gastrointest Endosc 69: S71-77.

460 19. Yan F, Wang S, Yang W, Goldberg SN, Wu H, Duan WL, Deng Z T, Han H B, Zheng HR.

461 2017. Tumor-penetrating Peptide-integrated Thermally Sensitive Liposomal Doxorubicin

462 Enhances Efficacy of Radiofrequency Ablation in Liver Tumors. Radiology 285:462-471.

463 20. Cho HJ, Lee SJ, Park SJ, Paik CH, Lee SM, Kim S, Lee YS. 2016. Activatable iRGD-

464 based peptide monolith: Targeting, internalization, and fluorescence activation for precise tumor

465 imaging. J Control Release237:177-184.

466 21. Maeda H. 2012. Vascular permeability in cancer and infection as related to macromolecular 467 drug delivery, with emphasis on the EPR effect for tumor-selective drug targeting. Proceedings 468 of the Japan Academy. Series B, Physical and biological sciences 88: 53-71. 
469 22. Kinoshita R, Ishima Y, Chuang VTG, Nakamura H, Fang J, Watanabe H, Shimizu T, 470 Okuhira K, Ishida T, Maeda H, Otagiri M, Maruyama T. 2017. Improved anticancer effects of 471 albumin-bound paclitaxel nanoparticle via augmentation of EPR effect and albumin-protein 472 interactions using S-nitrosated human serum albumin dimer. Biomaterials 140:162-169. 


\section{Figure 1}

Image of the LSPMbs obtained by transmission electron microscope.

Figure 1 Image of the LSPMbs obtained by transmission electron microscope. The LSPMbs present round shaped on a sectional view, with different size, without aggregation.

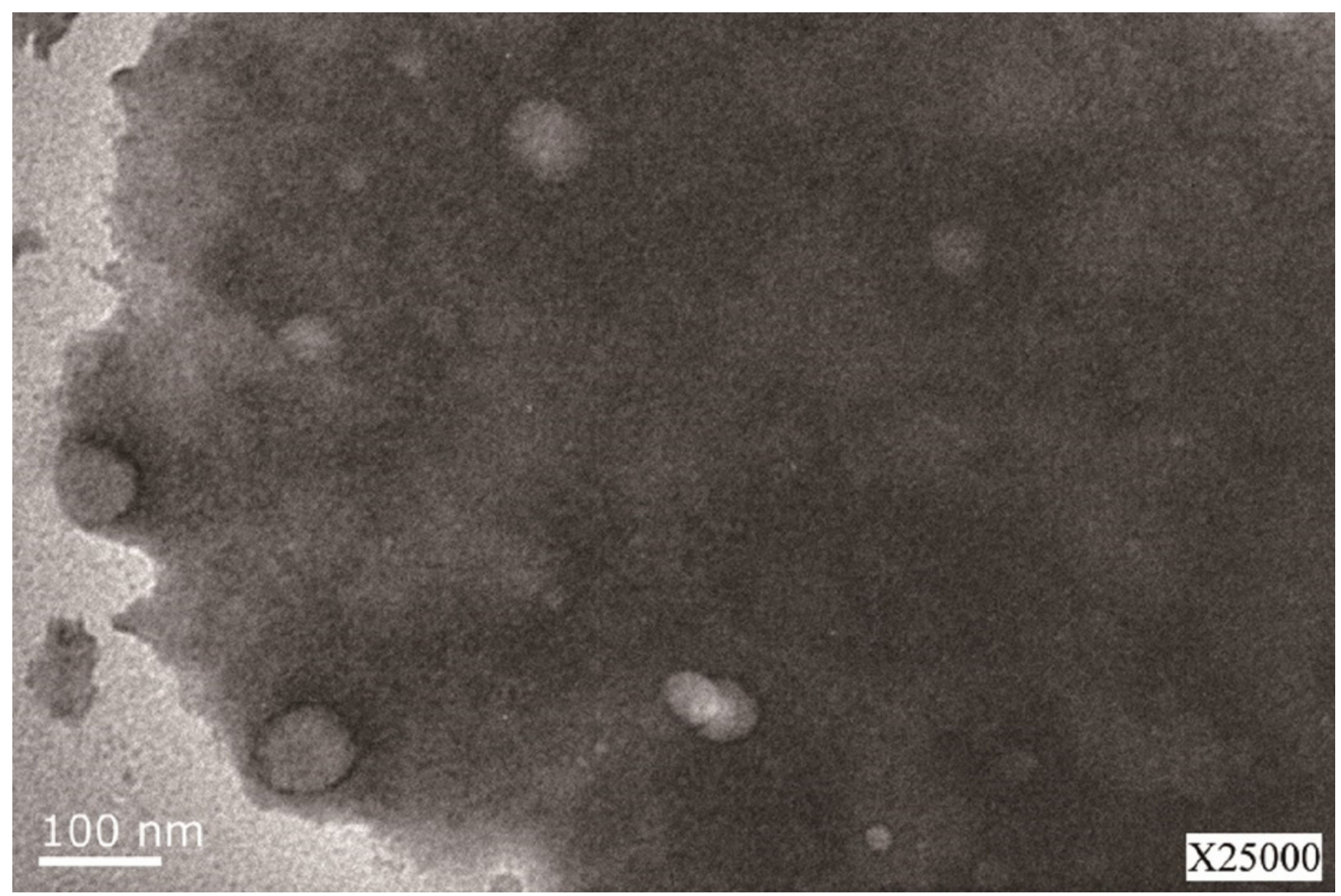


Figure 2

Size and distribution of LSPMbs

Figure 2 Size of LSPMbs are $380.9 \pm 176.5 \mathrm{~nm}$, and the determination results show "Good".

Size (d.n... \% Intensity: St Dev (d.n...

Z-Average (d.nm): 240.6

Pdl: 0.245

Intercept: 0.852

Result quality Good
Peak 1: $\quad 317.6$

Peak 2: $\quad 0.000$

Peak 3: $\quad 0.000$
100.0

0.0

0.0
175.7

0.000

0.000

\section{Size Distribution by Intensity}

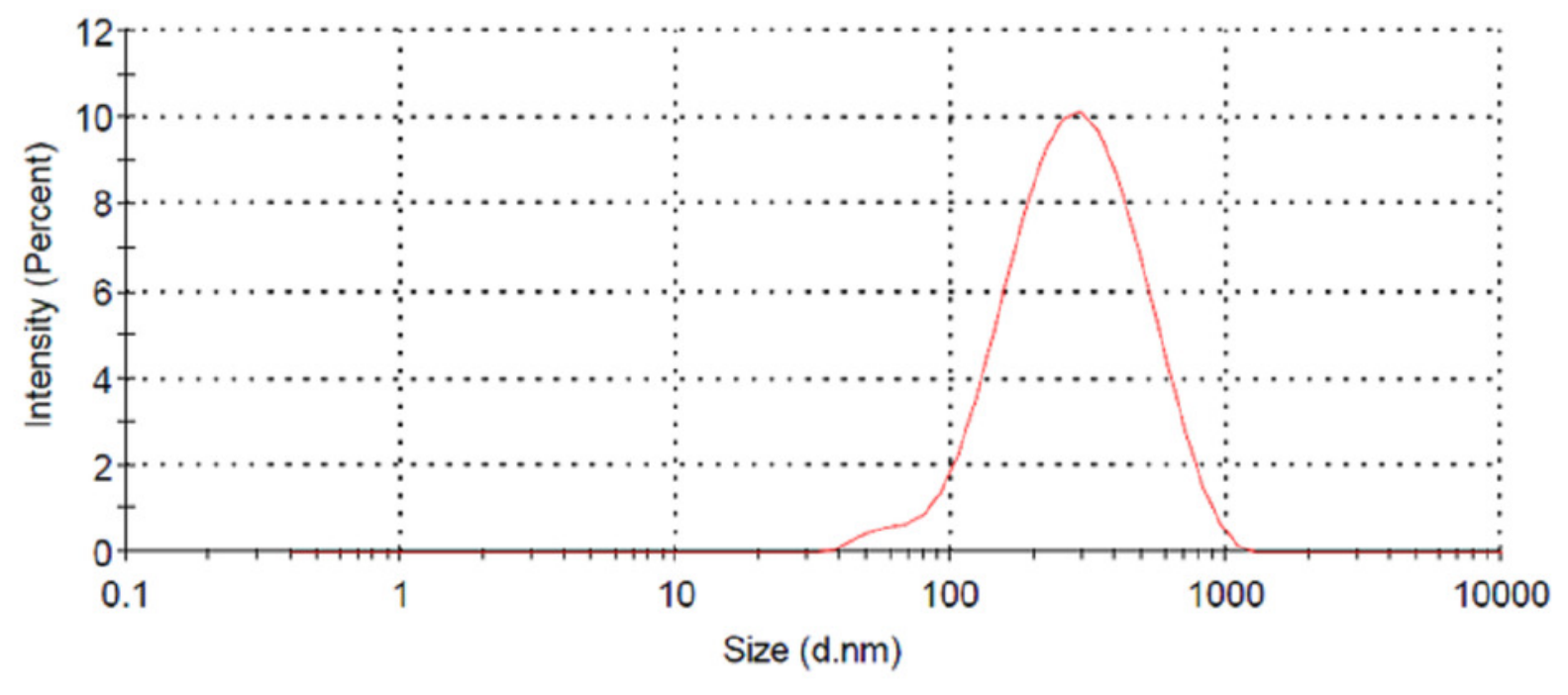


Figure 3

Zea potential and distribution of LSPMbs

Figure 3 Zea potential of LSPMbs was $-51.4 \pm 10.4 \mathrm{mV}$, and the determination results show "Good".

Mean $(\mathrm{mV}) \quad$ Area $(\%) \quad$ St $\operatorname{Dev}(\mathrm{mV})$

Zeta Potential (mV): -48.5

Zeta Deviation $(\mathrm{mV}): 7.61$

Conductivity (mS/cm): 1.30

Result quality Good
Peak 1: $\quad-48.5$

Peak 2: 0.00

Peak 3: 0.00
100.0

0.0

0.0 7.61 0.00 0.00

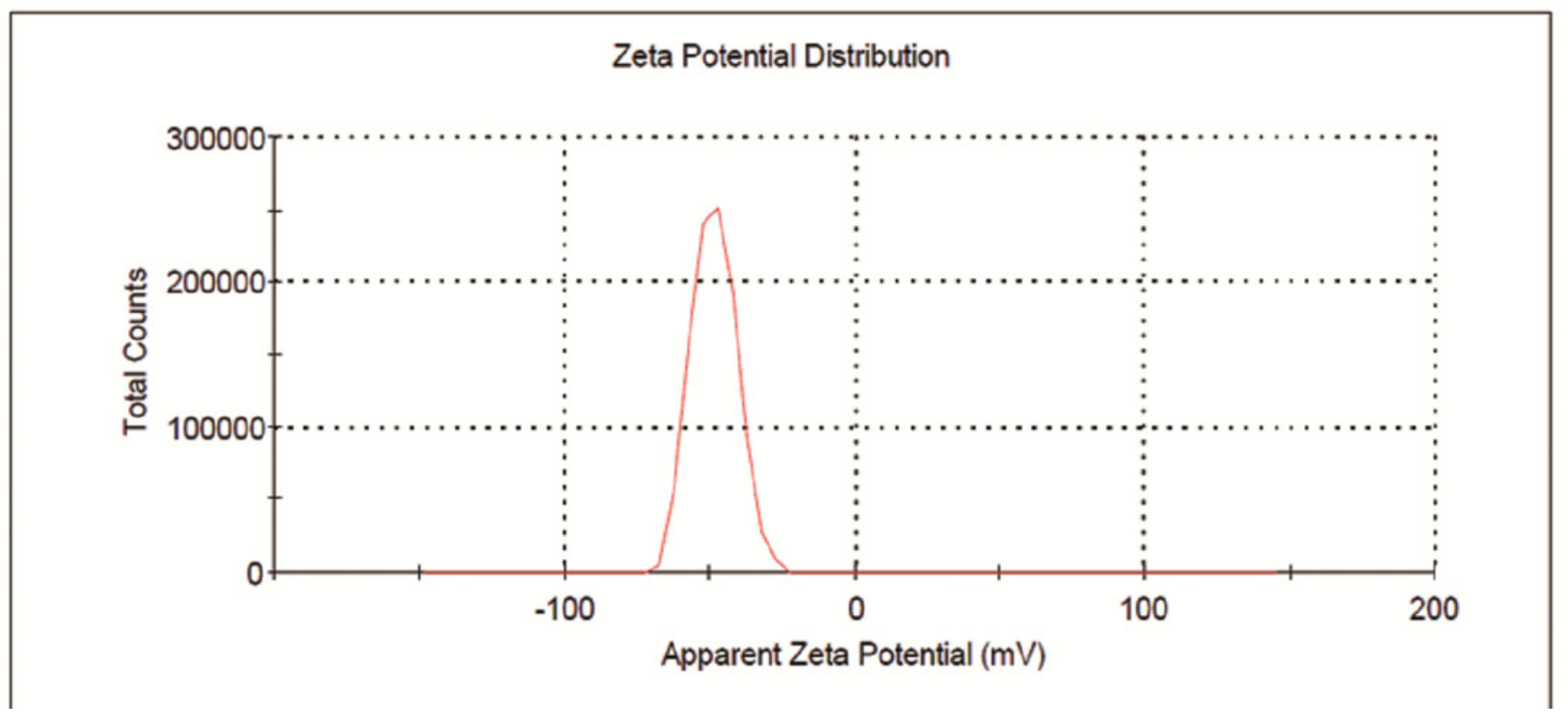


Figure 4

Figure 4

Figure 4 MTT assay for cytotoxicity showed the cell index had no significant differences among different LSPMbs concentrations at different time (all $P>0.05$ ).

\section{Cytotoxicity}

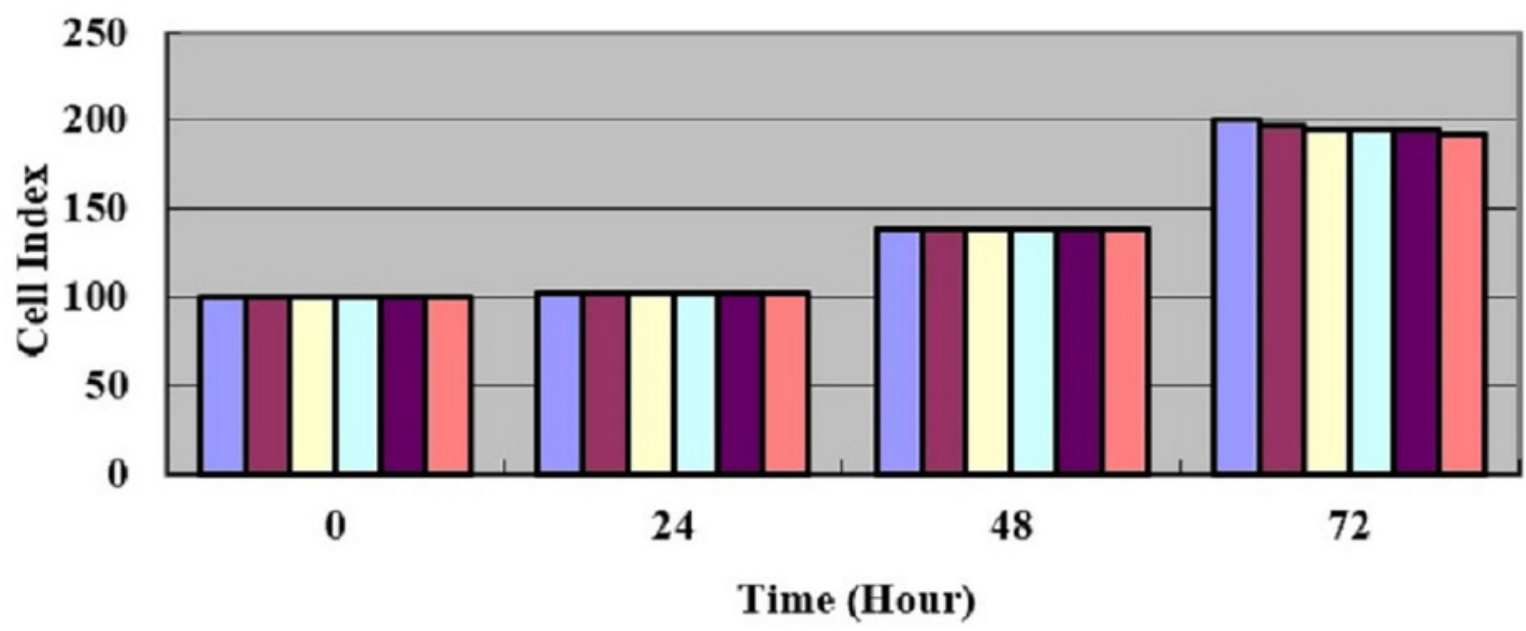

口0mg/mL; control $\square 2 \mathrm{mg} / \mathrm{mL}$

口5mg/mL

$\mathbf{\square} 10 \mathrm{mg} / \mathrm{mL}$

$\square 15 \mathrm{mg} / \mathrm{mL}$

$\square 20 \mathrm{mg} / \mathrm{mL}$

Time (Hour) 


\section{Figure 5}

Figure 5

Figure 5 Ultrasound images of the LSPMbs, SonoVue, degas deionized water, and air in the tubes. Figures $A$ and $D$ were obtained from LSPMbs and SonoVue of $1.6 \mathrm{mg} / \mathrm{mL}, B$ and $E$ were obtained from LSPMbs and SonoVue of $0.8 \mathrm{mg} / \mathrm{mL}$, and $\mathrm{C}$ and $\mathrm{F}$ were obtained from LSPMbs and SonoVue of $0.4 \mathrm{mg} / \mathrm{mL}$. Figure $\mathrm{G}$ was obtained from degas deionized water, and $\mathrm{H}$ was obtained from air. The echogenic intensity decreased with decreasing concentrations of LSPMbs, and degas deionized water; the echogenic intensity was strong at the interface between the air in the tube and the outside water, and the echogenicity in the tube was attenuated. 

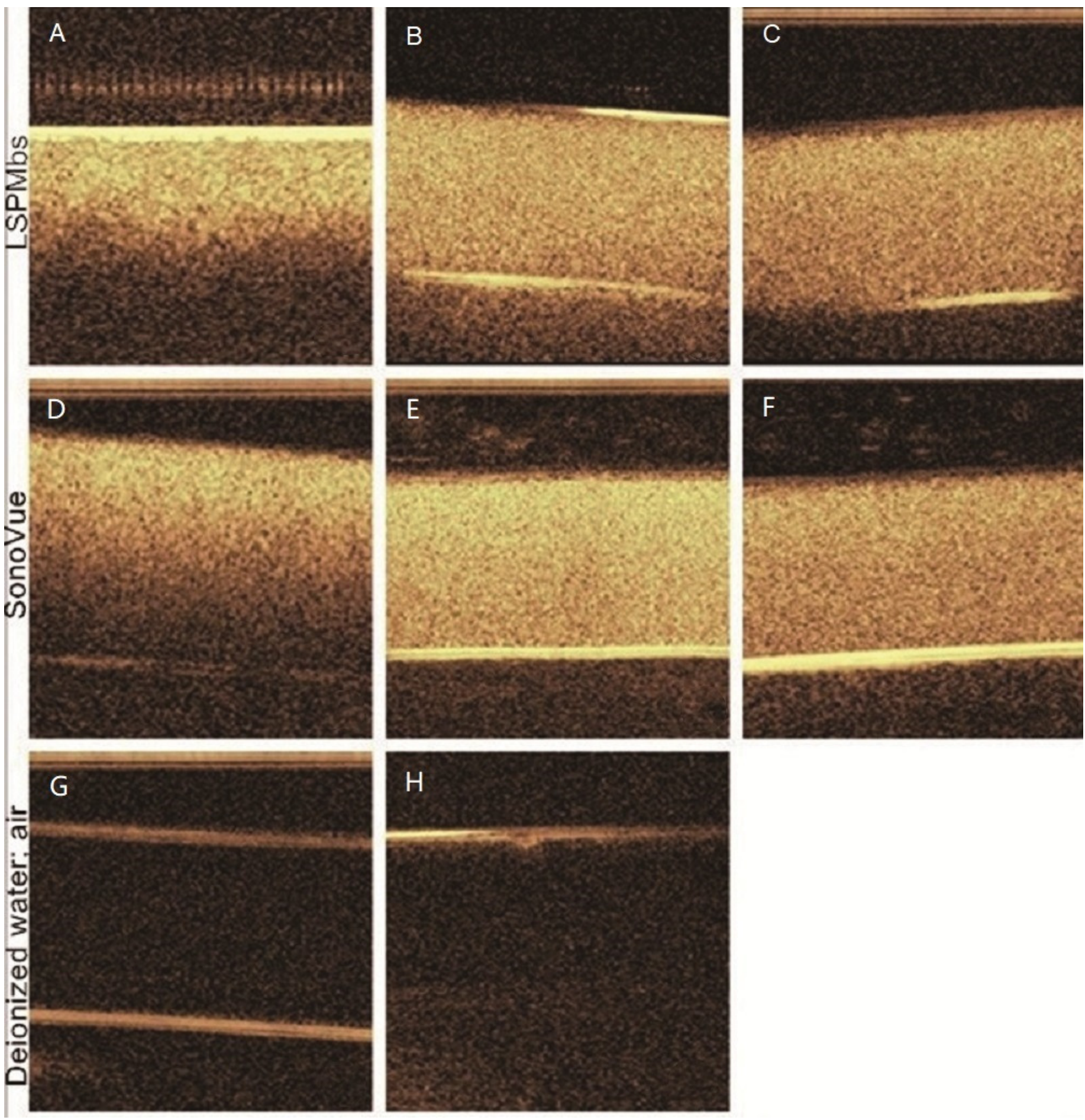


\section{Figure 6}

Figure 6

Figure 6 Image of GPC3 expression of Huh-7 cells obtained by confocal laser scanning microscope. The fluorescence on the cellular membrane appears intensive.

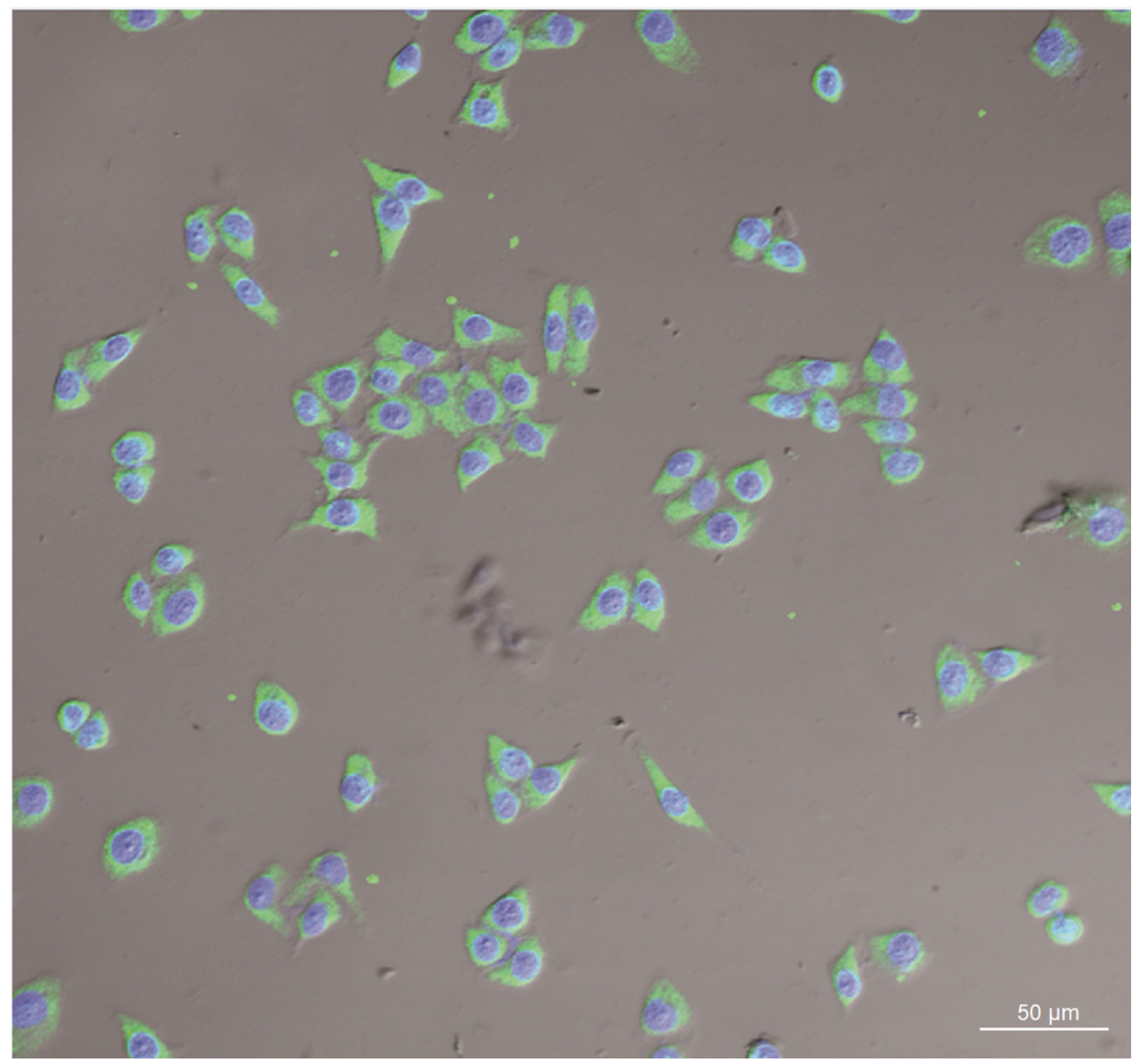




\section{Figure 7}

Figure 7

Figure 7 Images of Huh-7 cells incubated with Dil lablled LSPMbs and controls obtained by light microscope and confocal laser scanning microscope. (Images of A, E and I) Cells and Dil lablled LSPMbs and controls obtained from light microscope. (Images of B, F and J) Cell nucleus of Huh-7 cells presented blue after DAPI staining and being incubated with Dil lablled LSPMbs. (Images of C and D) LSPMbs with Dil staining combined with the membrane of Huh-7 cells presented red color fluorescence on image. (Images of G and H) LSGMbs presented a very similar appearance. (Images of $K$ and L) LSPMbs with Dil staining after blocked by synthesized peptide did not show red color fluorescence on the membrane of Huh-7 cells, indicating that LSPMbs had not combined with the membrane of Huh-7 cells. 

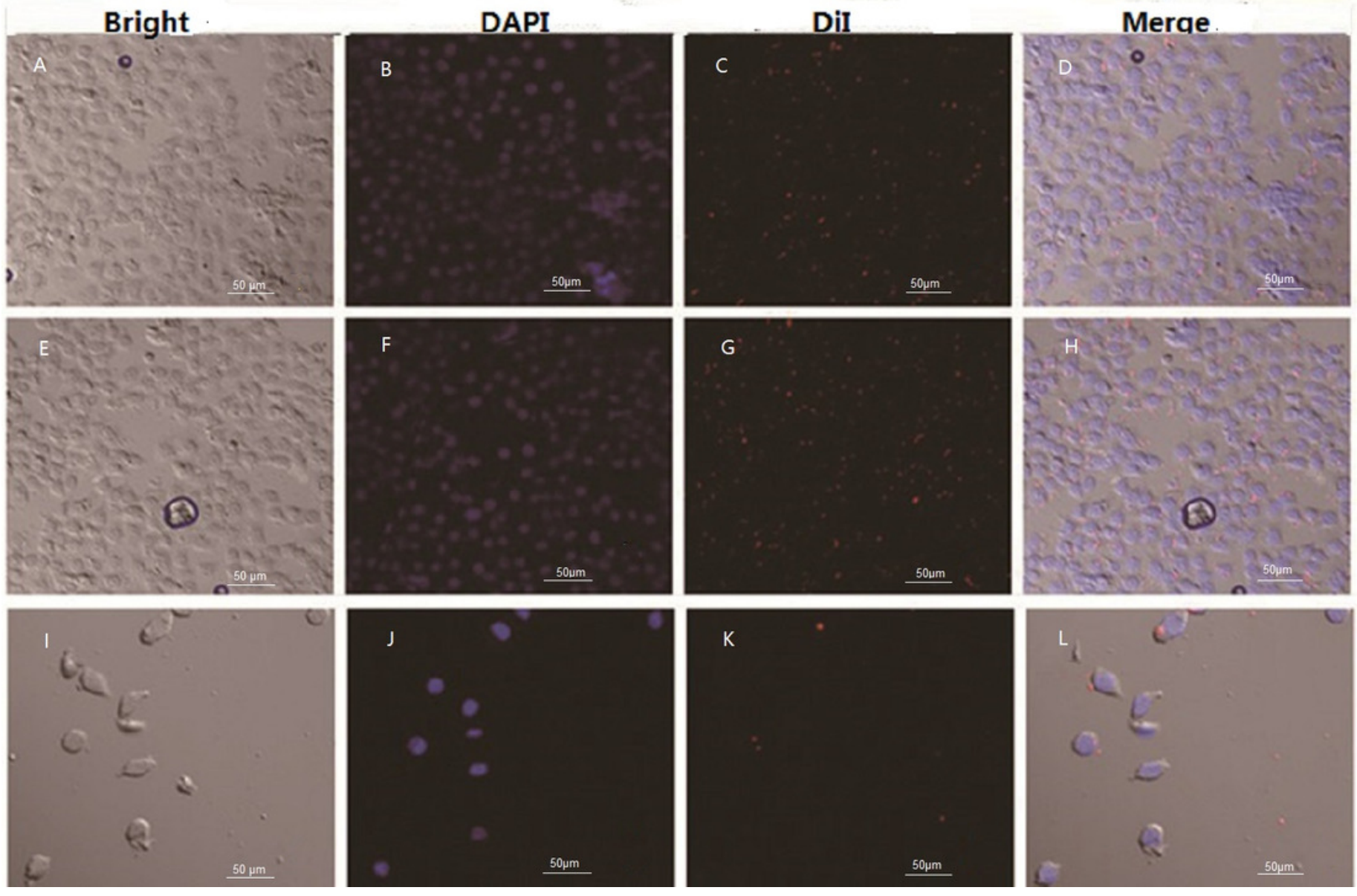


\section{Figure 8}

Figure 8

Figure 8 Row 1 is a study group, and rows 2-5 are control groups. (Images of A, E, I, M and Q) Images of all xenograft tumors presented a complex of isoechogenicity, hypoechogenicity and anechogenicity obtained by convention ultrasound. (Image B of LSGMbs) After administration of LSPMbs suspension, tumors presented hyper-enhancement in periphery and hypo-enhancement in center (necrosis) at two second. (Image C of LSGMbs) At 60 seconds, the tumor still presented hyper-enhancement in periphery and hypo-enhancement in center (necrosis). (Image D of LSGMbs) At 10 minutes, the tumor presented isoenhancement with central hypo-enhancement (necrosis). (Images F, G and H of LSGMbs) and (Images J, K and L of LSPMbs, after GPC3 blocking) and they presented similar enhancement patterns and sustain time as those in Huh-7 xenograft tumors, and there were no appreciable difference. (Images N, O and P of SonoVue) The xenograft tumors presented similar enhancement patterns and sustaining time as those using LSPMbs after administration of SonoVue suspension. (Images R, S and T of LS) The xenograft tumors did not present enhancement at 2, 20, and 60 seconds, and 10 minutes after administration of LS suspension. 

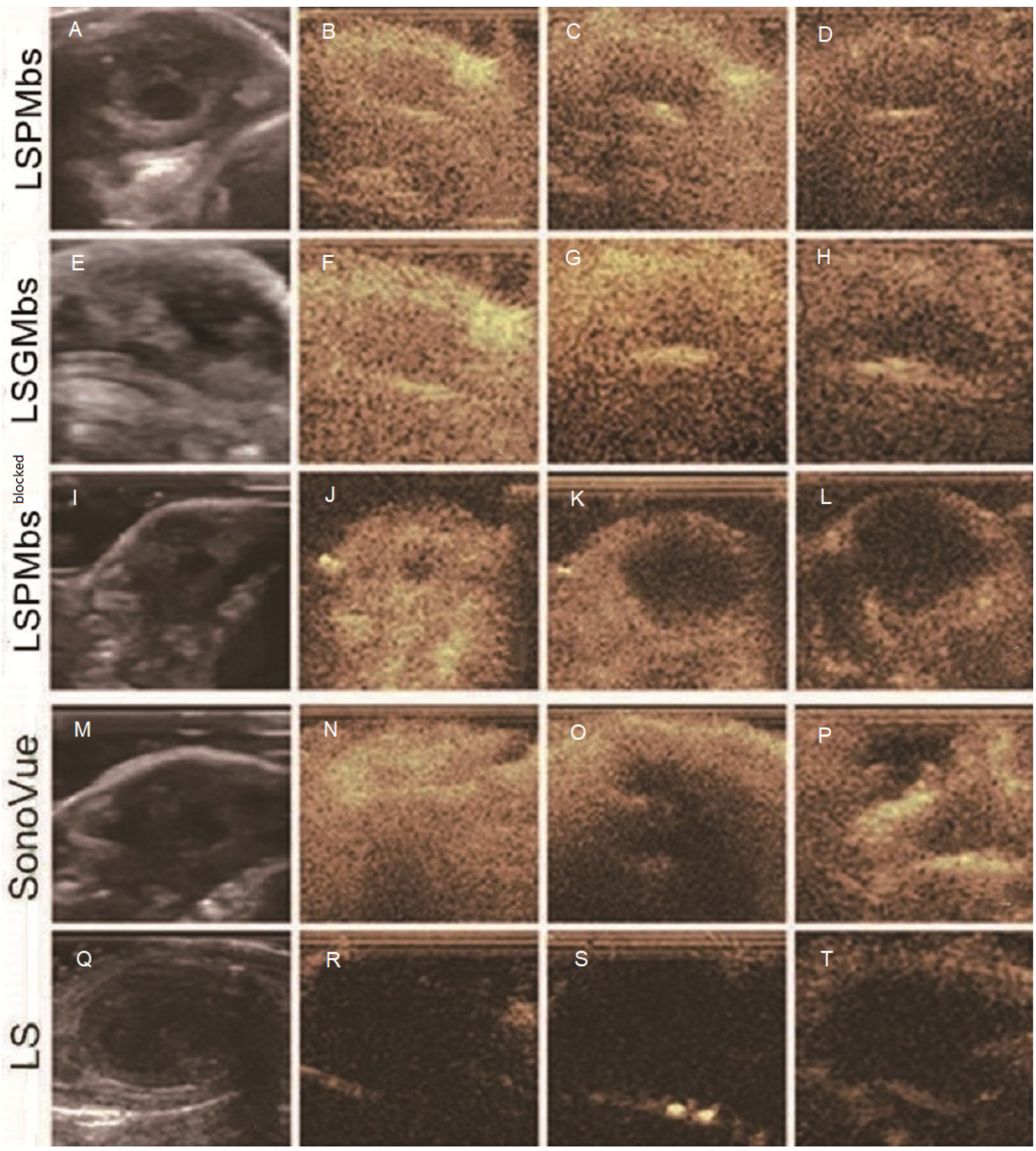


\section{Figure 9}

Figure 9

Figure 9 Image $A$ acquired at one minute, and image $B$ acquired six hours after the initial fluorescent imaging, the fluorescent signal intensity in the tumor area has no significant difference from other areas of the body expect the liver and spleen. Image C acquired 24 hours after injection, the fluorescent signal could not be visualized in the mice. Images D, E and $\mathrm{F}$ acquired from experiments of mice with Huh-7 xenograft tumors blocked using GPC3 antibody presented the same fluorescent imaging characteristics as those in the mice with Huh-7 xenograft tumors.
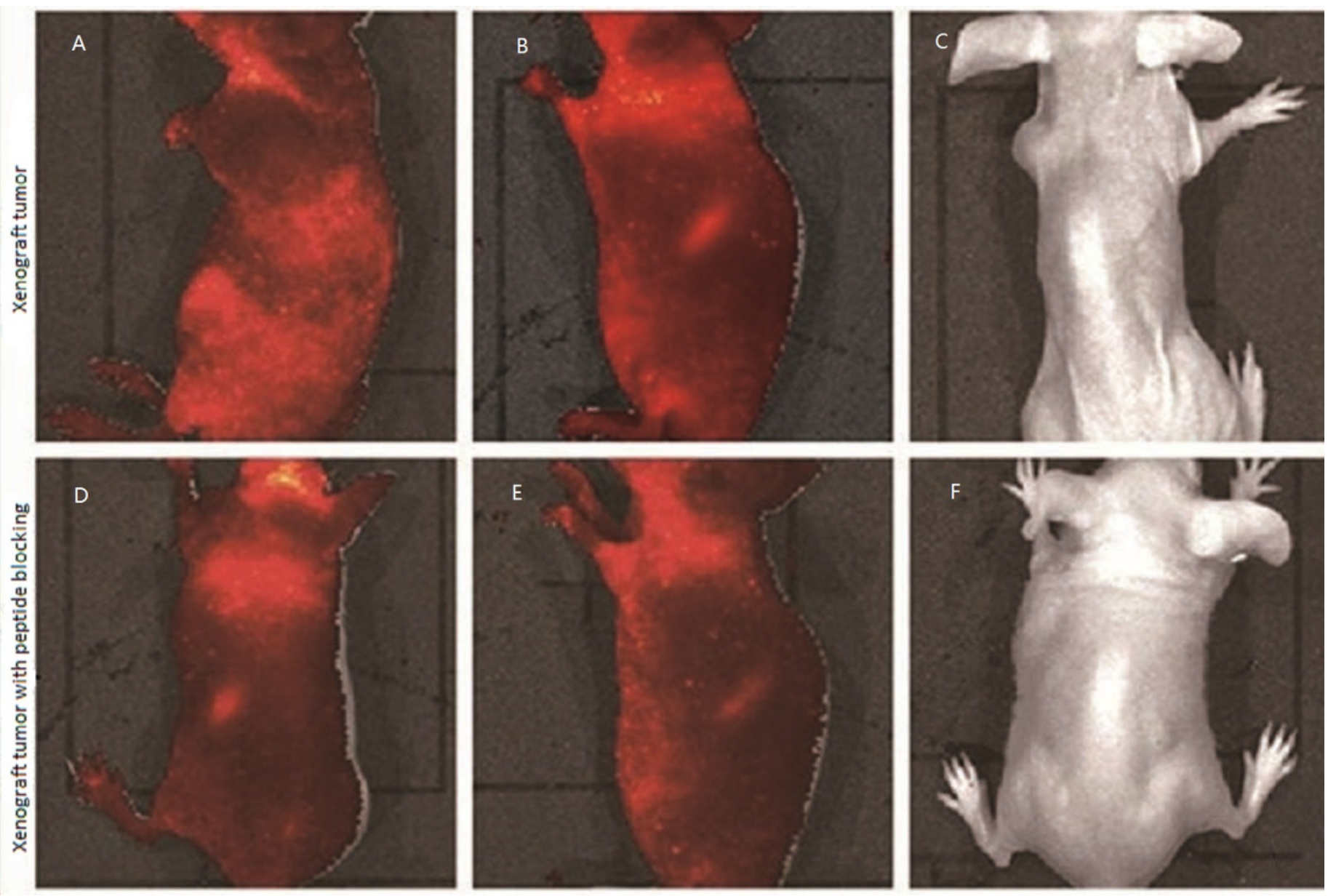


\section{Figure 10}

Fluorescence in the organs

Figure 10 There were fluorescence in the lungs and liver, and no fluorescence in the tumor, heart, spleen, and kidneys 24 hours after intravenous injection of Cy5.5 labelled LSPMbs, after animal sacrificed.

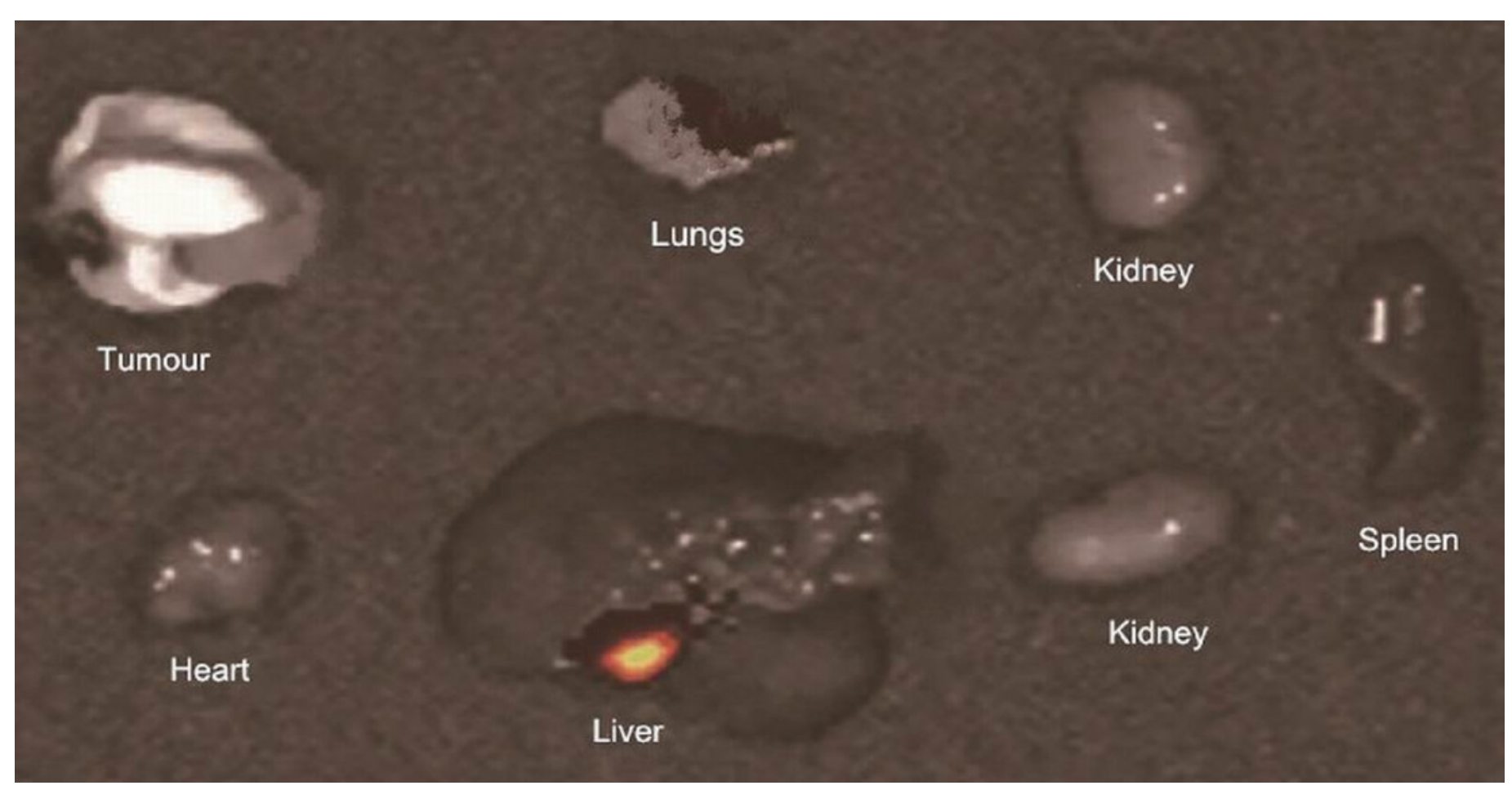

